\title{
Une Note sur les Topologies Linéaires
}

\author{
By Mohamed TABAÂ \\ Département de Mathématiques, Faculté des Sciences, Rabat, Maroc \\ (Communicated by Shokichi IYANAGA, M. J. A., May 12, 1997)
}

1. Introduction. Dans toute la suite, $A$ désigne un anneau commutatif unitaire intègre, $K$ son corps des fractions, $A^{\prime}$ sa clôture intégrale, $A^{*}$ sa quasi-clôture intégrale, $P(A)=\operatorname{Spec}(A)$ - $\{0\}, P_{1}(A)$ l'ensemble des idéaux premiers de hauteur 1 de $A$ et $\operatorname{Max}(A)$ l'ensemble de ses idéaux maximaux.

On désigne par $\tau_{A}$ la topologie linéaire sur $K$ qui admet pour système fondamental de voisinages de 0 les idéaux non nuls de $A$; si $p \in P(A)$, on notera $\tau_{p}$ la topologie $\tau_{A_{p}}$.

Rappelons deux définitions:

a) $A$ est dit $h$-semi-local si, pour tout idéal propre $a$ de $A$, l'anneau $A$ / $a$ est semi-local.

b) $A$ est dit topologiquement prüférien si, pour tout $p \in P(A)$, la topologie $\tau_{p}$ est définie par une valuation de $K$.

Dans cette Note nous montrons que, si $A$ est topologiquement prüférien $h$-semi-local, alors:

1) $A^{*}$ est un anneau de Prüfer $h$-semi-local de dimension $\leq 1$, et l'application $n \rightarrow n \cap A$ de $\operatorname{Max}\left(A^{*}\right)-\{0\}$ dans $P_{1}(A)$ est bijective; ce résultat généralise le théorème 1.8 de [1] et le corollaire 9 de [6];

2) Toute topologie de corps $A$-linéaire séparée non discrète sur $K$, est borne surpérieure de topologies $\tau_{m}(m \in \operatorname{Max}(A))$.

Comme application de ces deux résultats, nous obtenons une généralisation du théorème 2.14 de [1].

2. Résultats. Si $U$ est une partie de $K$, on désigne par $\mathfrak{f}_{U}$ le sous- $A$-module de $K$ formé des $x \in K$ tel que $x U \subset A$.

Lemme 1. Si $\tau_{A}$ est définie par une valuation de $K$, alors, pour tout sous- $A$-module $M$ de $K$ tel que $M \neq K$, on a $\mathfrak{f}_{M} \neq 0$.

Preuve. Soient $V$ un anneau de valuation de $K$ tel que $\tau_{A}=\tau_{V}$, et $x \in K-M$. Montrons que $\left(\mathfrak{f}_{V}\right)^{2} \subset x \mathfrak{f}_{M}$; en effet, on a $\left(\mathfrak{f}_{V}\right) V M \subset M$, donc $x V \not \subset\left(\mathfrak{f}_{V}\right) V M$, mais $V$ est un anneau de valuation, donc $\left(\mathfrak{f}_{V}\right) V M \subset x V$; d'où $\left(\mathfrak{f}_{V}\right)^{2} M \subset x A$, et par suite $\left(\mathfrak{f}_{V}\right)^{2} \subset x \mathfrak{f}_{M}$. Le lemme découle de cette inclusion et du fait que $\mathfrak{f}_{V} \neq 0$.

Corollaire 1. On suppose $\tau_{A}$ définie par une valuation de $K$. S'il existe un sous- $A$-module $M$ de $K$ ouvert pour une topologie d'anneau $\tau$ sur $K$ et tel que $M \neq K$, alors $\tau_{A}$ est moins fine que $\tau$.

Preuve. D'après le lemme précédent, il existe $d \neq 0$ tel que $d M \subset A$; le corollaire découle du fait que pour tout $a \neq 0$ l'homothétie $x \rightarrow a x$ est un homéomorphisme de $K$ sur lui-même.

On désigne par $L_{r}(A)$ (resp. $\left.L_{f}(A)\right)$ l'ensemble des topologies d'anneau (resp. de corps) $A$-linéaires séparées non discrètes sur $K$, et $\Re(A)$ le radical de $A$.

D'après [2, chap. $6 \S 5$ exer. 1$], \tau_{A} \in L_{r}(A)$, et $\tau_{A} \in L_{f}(A)$ si et seulement si $\Re(A) \neq 0$.

Le résultat suivant donne une caracterisation (topologique) des anneaux étudiés dans [4].

Proposition 1. Les propriétés suivantes sont équivalentes:

1) $\tau_{A}$ est définie par une valuation de $K$.

2) $L_{r}(A)$ est réduit à un seul élément.

3) $\Re(A) \neq 0$ et $L_{f}(A)$ est réduit à un seul élément.

Preuve. 1$) \Rightarrow 2)$. Si $\tau \in L_{r}(A)$, il est clair que $\tau$ est moins fine que $\tau_{A}$ et d'après le corollaire précédent $\tau_{A}$ est moins fine que $\tau$, d'où $\tau=$ $\tau_{A}$.

$2) \Rightarrow 3)$. Si $A \subset B$ est un sous-anneau local de $K$ tel que $B \neq K$, alors $\tau_{A}=\tau_{B}$ donc $\tau_{A} \in$ $L_{f}(A)$ et par suite $\Re(A) \neq 0$. La dernière assertion est claire.

$3) \Rightarrow 1)$. On a $\tau_{A} \in L_{f}(A)$, et d'après $[2$, chap. $6 \S 1 \mathrm{n}^{\circ} 2$ th. 2] il existe un anneau de valuation $V$ de $K$ tel que $A \subset V$ et $V \neq K$, d'où $\tau_{A}=\tau_{V}$.

De cette proposition découle immédiatement la proposition 1.5 de [1].

Remarque. Let anneaux vérifiant les propriétés équivalentes de la proposition précédente sont caractérisés dans ([2, chap. $6 \quad \S 5$ exer. 3b)],[9, prop. 1]) pour les anneaux noethériens, et dans [11, chap. 1 prop. 2] pour les anneaux de 
dimension 1 .

La proposition suivante est bien connue (cf. [4]); nous la démontrons directement.

Proposition 2. On suppose que $\tau_{A}$ est définie par une valuation de $K$. Si $A^{*} \neq K$, alors $A^{*}$ est un anneau de valuation de hauteur 1 , et la trace sur $A$ de son idéal maximal est le plus petit élément de $P(A)$ ordonné par inclusion.

Preuve. D'après le lemme 1 , si $A \subset B$ est un sous-anneau de $K$ tel que $B \neq K$, il existe $d \neq 0$ tel que $d B \subset A$, d'où $B \subset A^{*}$. Il en résulte, d'une part que $A^{*}$ est anneau de valuation de hauteur $1\left[2\right.$, chap. $6 \S 4 \mathrm{n}^{\circ} 5$ prop. 6], et d'autre part que si $q \in P(A)$ alors $A_{q} \subset A^{*}$, d'où si $p$ est la trace de l'idéal maximal de $A^{*}$, on a $p \subset q$.

On désigne par $V_{1}(A)$ l'ensemble des anneaux de valuation de hauteur 1 de $K$ contenant $A$.

Si $A$ est topologiquement prüférien, il résulte de la proposition 2 que l'application $V \rightarrow m_{V} \cap$ $A$, où $m_{V}$ est l'idéal maximal de $V$, de $V_{1}(A)$ dans $P_{1}(A)$, est bien définie.

Proposition 3 [1, prop. 1.7]. Si $A$ est topologiquement prüférien alors l'application $V \rightarrow m_{V}$ $\cap A$ de $V_{1}(A)$ dans $P_{1}(A)$ est bijective.

Preuve. D'après le lemme 1 de [10] et la proposition 2, l'application $p \rightarrow\left(A_{p}\right)^{*}$ de $P_{1}(A)$ dans $V_{1}(A)$, est bien définie; on vérifie facilement que les applications $V \rightarrow m_{V} \cap A$, et $p \rightarrow\left(A_{p}\right)^{*}$ sont réciproques l'une de l'autre.

Théorème 1. $\mathrm{Si} A$ est topologiquement prüférien et $h$-semi-local, alors: $\leq 1$

1) $A^{*}$ est un anneau de Prüfer de dimension

2) l'application $n \rightarrow n \cap A$ est une bijection de $\operatorname{Max}\left(A^{*}\right)-\{0\}$ sur $P_{1}(A)$.

Preuve. Montrons 1). On suppose $A^{*} \neq K$. D'après [7, lemma 2.2], on a $A^{*}=\bigcap_{m \in \operatorname{Max}(A)}\left(A_{m}\right)^{*}$ car la famille $\left(A_{m}\right)_{m \in \operatorname{Max}(A)}$ satisfait à la condition FC. Si $E$ est l'ensemble des $m \in \operatorname{Max}(A)$ tels que $\left(A_{m}\right)^{*} \neq K$, alors $A^{*}=\bigcap_{m \in E}\left(A_{m}\right)^{*}$. D'après la proposition 2 , pour tout $m \in E,\left(A_{m}\right) *$ est un anneau de valuation de hauteur 1 , et comme la famille $\left(\left(A_{m}\right)^{*}\right)_{m \in E}$ satisfait à la condition FC. $A^{*}$ est donc un anneau de caractère fini et de type réel. Soit $n \in \operatorname{Max}\left(A^{*}\right)$; d'après $[2$, chap. $7 \S 1$ exer. 26.d)] $\left(A^{*}\right)_{n}$ est un anneau de caractère fini et de type réel; donc il est complètement intég- ralement clos $\left[2\right.$, chap. $6 \S 4 \mathrm{n}^{\circ} 5$ cor. de la prop. 9]. Mais, en vertu de la proposition $1, \tau_{n}$ est définie par une valuation de $K$; il résulte donc, de la proposition 2 et [2, chap. $6 \S 4 \mathrm{n}^{\circ} 5$ prop.6], que $\left(A^{*}\right)_{n}$ est un anneau de valuation de dimension 1 . Donc $A^{*}$ est un anneau de Prüfer $h$-semi-local de dimension 1 . L'assertion 2 découle de l'assertion précédente et de la proposition 3 .

Corollaire 2 [6, cor. 9]. Si $A$ est un anneau de Prüfer $h$-semi-local alors $A^{*}$ est un anneau de Prüfer de dimension $\leq 1$.

Remarques. 1) Posons $A^{\prime \prime}=\bigcap_{V \in V_{1}(A)} V$; dans la démonstration de l'assertion i) du théorème 1.8 de [1], l'auteur utilise le fait que, si $A$ est topologiquement prüférien, $h$-semi-local et $P_{1}(A) \neq \varnothing$, alors pour tout $p^{\prime \prime} \in P\left(A^{\prime \prime}\right)$ il existe $V \in$ $V_{1}(A)$ tel que $A^{\prime \prime}{ }_{p^{\prime \prime}} \subset V$; ce qui est incorrect, comme le montre l'exemple suivant:

Soient $v$ une valuation d'un corps $k$ dont l'anneau est sans idéal premier de hauteur $1[5$, exemple 19.12], $K=k(X)$, où $X$ est une indéterminée, $v_{1}$ l'unique valuation de $K$ prolongeant $v$, telle que $v_{1}(X)=0, V_{1}$ son anneau et $V_{2}=$ $k[X]_{(X)}$. Posons $A=V_{1} \cap V_{2}$. D'après [2, chap. $6 \S 10 \mathrm{n}^{\circ} 1$ prop. 2], $V_{1}$ est un anneau de valuation sans idéal premier de hauteur 1 , et $V_{2}$ est un anneau de valuation discrète; notons $m_{i}$ l'idéal maximal de $V_{i}$ et posons $p_{i}=m_{i} \cap A$. D'après [2, chap. $6 \S 7 \mathrm{n}^{\circ} 1$ prop. 1 et 2$] A$ est un anneau de Prüfer semi-local d'idéaux maximaux $p_{1}, p_{2}$, $P_{1}(A)=\left\{p_{2}\right\}$, mais il n'existe aucun anneau de valuation de hauteur 1 de $K$ contenant $A_{P_{1}}=V_{1}$ [2, chap. $6 \S 4 \mathrm{n}^{\circ} 11$ prop. 1$]$.

2) La première assertion du théorème précédent entraîne que l'assertion iii) du théorème 1.8 de [1] est valable sans hypothèse supplémentaire.

Corollaire 3. $\mathrm{Si} A$ est topologiquement prüférien $h$-semi-local, alors $A$ est complètement intégralement clos si et seulement si $A$ est un anneau de Prüfer de dimension 1.

Preuve. La condition nécessaire résulte $\mathrm{du}$ théorème précédent. La condition suffisante découle de [5, th. 23.4].

La démonstration du théorème 2 utilise le

Lemme 2. Soit $a$ un idéal de $A$ tel que $a \neq$ $A$. Si $E$ est l'ensemble des $m \in \operatorname{Max}(A)$ tels que $a \subset m$, alors $(1+a)^{-1} A=\bigcap_{m \in E} A_{m}$.

Prenve. L'inclusion $\subset$ est claire, reste à 
montrer l'autre; soit donc $x \in \bigcap_{m \in E} A_{m}$; pour tout $m \in E \quad$ on $\quad$ a $\quad \mathfrak{f}_{x} \cap A \not \subset m ;$ donc $a+\left(\mathfrak{f}_{x} \cap\right.$ $A)=A$, d'où $1=\boldsymbol{a}+\boldsymbol{b}$, avec $\boldsymbol{a} \in \boldsymbol{a}$ et $\boldsymbol{b} \in \mathfrak{f}_{x} \cap$ $A$, on en déduit que $x=\frac{b x}{1-a} \in(1+a)^{-1} A$.

On dira que $A$ vérifie la propriété $(M)$ si toute topologie $\tau \in L_{f}(A)$ est borne supérieure de topologies $\tau_{m}(m \in \operatorname{Max}(A))$.

La démonstration du résultat suivant est une adaptation de celle que nous avons donnée dans [11] pour $A$ de dimension 1.

Théorème $2[1$, th. 1.3]. Si $A$ est topologiquement prüférien $h$-semi-local alors $A$ vérifie la propriété $(M)$.

Preuve. Soient $\tau \in L_{f}(A)$ et $F$ l'ensemble des $m \in \operatorname{Max}(A)$ tels que $\tau_{m}$ soit moins fine que $\tau$. Montrons que $\tau=\sup _{m \in F} \tau_{m}$. Pour cela il suffit de montrer que $\tau$ est moins fine que $\sup _{m \in F} \tau_{m}$. Soit donc $M$ un sous- $A$-module de $K$ ouvert pour $\tau$. L'application $(x, y) \rightarrow x y^{-1}$ de $K \times(K-\{0\})$ dans $K$ est continue en $(0,1)$, donc il existe un sous- $A$-module $N$ de $K$ ouvert pour $\tau$ tel que $N$ $\subset M, 1 \notin N$ et $N(1+N)^{-1} \subset M$. On pose $a=$ $N \cap A$ et soient $\boldsymbol{a} \in \boldsymbol{a}, \boldsymbol{a} \neq 0$ et $E$ l'ensemble des $m \in \operatorname{Max}(A)$ tels que $a \subset m$. On a bien $\boldsymbol{a}$ $(1+a)^{-1} A \subset M$, et d'après le lemme précédent on a $(1+a)^{-1} A=\bigcap_{m \in E} A_{m}$, d'où $\bigcap_{m \in E} \boldsymbol{a} A_{m} \subset M$.

D'autre part, $A$ est $h$-semi-local donc $E$ est fini; et pour tout $m \in E, \tau_{m}$ est définie par une valuation de $K$ et $1 \notin N_{m}$, donc, d'après le corollaire $1, \tau_{m}$ est moins fine que $\tau$. On en déduit que $M$ est ouvert pour la topologie $\sup _{m \in F} \tau_{m}$, ce qui achève la démonstration du théorème.

Remarques. 1) $\mathrm{Si}$ on applique le théorème précédent à la topologie linéaire $\tau^{A}$, définie dans [9], qui admet pour système fondamental de voisinages de 0 les sous- $A$-modules de $K$, de la forme $(1+a)^{-1} a$, où $a$ est un idéal propre de $A$, on retrouve le lemme 13 de [8].

2) Le théorème précédent et sa réciproque sont énoncés dans [9, th. 2 et prop. 1] pour les anneaux noethériens (cf. aussi [12, chap. 12 th. $5]$ ), et démontrés par l'auteur dans $[11$, chap. 1 th. 1] pour les anneaux de dimension 1 .

$3)$ D'après le corollaire 2.3 de [1], si $A$ vérifie la propriété $(M)$ et la condition $(*)$ définie dans [1], alors il est de dimension 1 (notons qu'en vertu du lemme 1 de [10], si $A$ est de dimension
1 alors il vérifie la condition $(*))$.

4) $A$ peut vérifier la propriété de la borne supérieure définie dans [1] sans qu'il soit $h$-semi-local. On prend $A=k[Y]+X k[X$, $Y]_{(X)}$, où $k$ est un corps, $X$ et $Y$ sont des indéterminées. $A^{*}=k[X, Y]_{(X)}$ est un anneau de valuation discrète, et $\mathfrak{f}_{A^{*}} \neq 0$ donc, en vertu de la proposition $1, L_{f}(A)$ est réduit à un seul élément et par suite $A$ vérifie la propriété de la borne supérieure; mais $A / X k[X, Y]_{(X)} \cong k[Y]$, donc $A$ n'est pas $h$-semi-local (notons que, d'après l'isomorphisme précédent et la proposition $2, A$ est de dimension 2).

Lemme 3. Soit $B$ un sur-anneau de $A$, intègre et tel que $B$ soit une $A$-algèbre finie. Si $A$ est $h$-semi-local, il en est de même de $B$.

Preuve. Soient $b$ un idéal propre de $B$ et $a$ $=b \cap A$; posons $\bar{A}=A / a, \bar{B}=B / b$, et désig. nons par $\varphi: \bar{A} \rightarrow \bar{B}$ l'homomorphisme déduit de l'inclusion $A \subset B$ par passage aux quotients et ${ }^{a} \varphi: \operatorname{Spec}(\bar{B}) \rightarrow \operatorname{Spec}(\bar{A})$ l'application associée. $a$ est un idéal propre de $A$ et $\bar{B}$ est une $\bar{A}$-algèbre finie. Le lemme découle donc de $[2$, chap. $5 \S 2$ $\mathrm{n}^{\circ} 2$ prop. 3] puisque, $\bar{A}$ est semi-local et, d'après [2, Chap. $5 \quad \S 2 \quad \mathrm{n}^{\circ} 1$ prop. 1], on a $\left({ }^{a} \varphi\right)^{-1}$ $(\operatorname{Max}(\bar{A}))=\operatorname{Max}(\bar{B})$. $P(A)$.

$A$ est dit divisé si $p=p A_{p}$, pour tout $p \in$

La proposition suivante généralise le théorème 2.14 de [1].

Proposition 4. On suppose $A h$-semi-local, localement divisé et localement cohérent. Si $A^{\prime}$ est une $A$-algèbre finie, alors:

1) si $\tau \in L_{f}(A)$ alors $\tau \in L_{f}\left(A^{\prime}\right)$ et $\tau$ vérifie la propriété $(M)$ dans $L_{f}\left(A^{\prime}\right)$;

2) $A^{*}$ est un anneau de Prüfer $h$-semi-local de dimension $\leq 1$.

Preuve. On a $\mathfrak{f}_{A^{\prime}} \neq 0$, donc si $\left(M_{\lambda}\right)_{\lambda}$ est un système fondamental de voisinages de 0 pour $\tau$, formé de sous- $A$-modules de $K$, alors $\left(A^{\prime} M_{\lambda}\right)_{\lambda}$ est aussi un système fondamental de voisinages de 0 pour $\tau$; d'où la première assertion de 1 ). D'autre part, d'après le corollaire 3.4 de [3], $A^{\prime}$ est un anneau de Prüfer, et en vertu du lemme précédent $A^{\prime}$ est $h$-semi-local; donc la deuxiéme assertion de 1) découle du théorème précédent, et l'as. sertion 2) découle du corollaire 2 , puisque $A^{*}=$ $\left(A^{\prime}\right)^{*}$.

Sous les hypothèses de la proposition précédente $A$ ne vérifie pas nécessairement $(M)$. 
On prend pour $A$ l'anneau intègre donné dans [2, chap. $5 \S 3$ exer. $5 c$ )]. $A$ est un anneau local noethérien de dimension 1 , sa clôture intégrale est une $A$-algèbre finie ayant deux idéaux maximaux; d'après les propositions 1 et $2, A$ ne vérifie pas la propriété $(M)$.

\section{Références}

[1] D. Abouabdillah: Topologies de corps $A$ linéaires. Pac. J. Math., 107, 257-266 (1983).

[2] N. Bourbaki: Algèbre commutative. Masson, Paris (1985).

[ 3 ] D. E. Dobbs: Coherence, ascent of going-down, and pseudeo-valuation domains. Houston J. Math., 4, 551-567 (1978).

[4] D. E. Dobbs and R. Fedder: Conducive domains. J. Algebra, 86, 494-510 (1984).

[5] R. Gilmer: Multiplicative Ideal Theory. Queen's
Papers in Pure and Appl. Math., Queen's University, Toronto (1992).

[6] R. Gilmer and W. Heinzer: On the complete integral closure of an integral domain. J. Aust. Math. Soc., 6, 351-361 (1966)

[ 7 ] W. Heinzer, J. Ohm, and R. Pendleton: On integral domains of the form $\cap R_{p}, P$ minimal. J. Reine Angew. Math., 241, 147-159 (1970).

[8] J. Mockor: The completion of Prüfer domains. Proc. Amer. Math. Soc., 221, 1-10 (1977).

[9] A. Jebli: Corps des fractions et topologies artiniennes. C. R. Acad. Sci. Paris, sér. A, 278, 973-976 (1974).

[10] P. Ribenboim: Sur une conjecture de Krull en théorie des valuations. Nagoya Math. J., 9, 87-97 (1955).

[11] M. Tabaâ: Sur une question de compacité linéaire. Thèse de $3^{\text {eme }}$ cycle, Rabat (1978).

[12] W. Wieslaw: Topological fields. Wroclaw (1982). 